\title{
Roles of saliency and set size in ensemble averaging
}

\author{
Aleksei U. lakovlev ${ }^{1}$ (D) $\cdot$ Igor S. Utochkin ${ }^{1}$
}

Published online: 24 July 2020

(C) The Psychonomic Society, Inc. 2020

\begin{abstract}
Ensemble statistics are often thought of as a reliable impression of numerous items despite limited capacities to consciously represent each individual. However, whether all items equally contribute to ensemble summaries (e.g., mean) and whether they might be affected by known limited-capacity processes, such as focused attention, is still debated. We addressed these questions via a recently described "amplification effect," a systematic bias of perceived mean (e.g., average size) towards the more salient "tail" of a feature distribution (e.g., larger items). In our experiments, observers adjusted the mean orientation of sets of items varying in set size. We made some of the items more salient or less salient by changing their size. While the whole orientation distribution was fixed, the more salient subset could be shifted relative to the set mean or differ in range. We measured the bias away from the set mean and the standard deviation $(S D)$ of errors, as it is known to reflect the physical range from which ensemble information is sampled. We found that bias and $S D$ changes followed the shifts and range changes in salient subsets, providing evidence for amplification. However, these changes were weaker than those expected from sampling only salient items, suggesting that less salient items were also sampled. Importantly, the $S D$ decreased as a function of set size, which is only possible if the number of sampled elements increased with set size. Overall, we conclude that orientation summary statistics are sampled from an entire ensemble and modulated by the amplification effect of attention.
\end{abstract}

Keywords Ensemble perception $\cdot$ Attention $\cdot$ Saliency $\cdot$ Sampling $\cdot$ Amplification effect

\section{Introduction}

It has been previously demonstrated that people can efficiently extract different types of statistical information from briefly presented multiple items. Summary statistics such as mean and variance can be extracted about various features of individual items from basic sensory dimensions, like orientation (Alvarez \& Oliva, 2009; Dakin \& Watt, 1997; Morgan, Chubb, \& Solomon, 2008; Parkes, Lund, Angelucci, Solomon, \& Morgan, 2001; Suárez-Pinilla, Seth, \& Roseboom, 2018), size (Ariely, 2001; Chong \& Treisman, 2003; Khvostov \& Utochkin, 2019; Tokita, Ueda, \& Ishiguchi, 2016), and color (Bronfman, Brezis, Jacobson, \& Usher, 2014; de Gardelle \& Summerfield, 2011; Maule \& Franklin, 2015), to quite complex and high-level dimensions, like facial expression (Haberman, Lee, \& Whitney, 2015;

Aleksei U. Iakovlev

auiakovlev@gmail.com

1 Psychology Department, HSE University, 4-2, Armyansky per., Moscow, Russia 101000
Haberman \& Whitney, 2007) or animacy (Leib, Kosovicheva, \& Whitney, 2016). Interestingly, the efficiency and accuracy of such ensemble representation does not suffer (Ariely, 2001; Chong \& Treisman, 2005a; Fouriezos, Rubenfeld, \& Capstick, 2008; Haberman, Harp, \& Whitney, 2009; Haberman \& Whitney, 2009), or even benefits (Chong, Joo, Emmanouil, \& Treisman, 2008; Robitaille \& Harris, 2011), from increasing set size, whereas our ability to report individual items quickly degrades with set size (Ariely, 2001; Haberman et al., 2009; Haberman \& Whitney, 2007, 2009). This important peculiarity makes many researchers think of ensemble summary statistics as of an important tool our visual system uses to deal with severe capacity limits of deep object processing (Alvarez, 2011; Bronfman et al., 2014; Chong et al., 2008; Cohen, Dennett, \& Kanwisher, 2016; Fischer \& Whitney, 2011; Rosenholtz, 2016, 2011).

However, there is still no strong consensus regarding the computational capacity of a mechanism beyond this efficient ensemble representation. According to one theory, ensemble summary statistics are accomplished via parallel exhaustive processing that has no restrictions on the number of items taken into account (Ariely, 2001; Attarha, Moore, \& Vecera, 2014; Baek \& Chong, 2020; Chong et al., 2008; Chong \& 
Evans, 2011; Chong \& Treisman, 2005a; Utochkin \& Tiurina, 2014). Presumably, a low-resolution but high-capacity mode of processing involving globally distributed attention can be a proper mechanism for that (Alvarez, 2011; Chong et al., 2008; Chong \& Treisman, 2005a; Treisman, 2006). An opposite theory argues that ensemble summary representations are supported by a limited-capacity mechanism associated with the bottleneck of focused attention and working memory. This theory suggests that only a small subset of items is sampled and integrated to accomplish proxy statistics for the entire ensemble (Allik, Toom, Raidvee, Averin, \& Kreegipuu, 2013; Maule \& Franklin, 2016; Myczek \& Simons, 2008; Solomon, 2010). Based on different model estimates, it was suggested that the visual system might integrate a fixed number of items (Maule \& Franklin, 2016; Myczek \& Simons, 2008) or a flexible number of items depending on the number of observed items like the square root of presented items (Dakin, 2001; Gorea, Belkoura, \& Solomon, 2014; Solomon, 2010; Whitney \& Yamanashi Leib, 2018) or about a half of them (Allik et al., 2013).

Unlike the theories suggesting parallel exhaustive processing of all items, the limited-capacity models should explain rules used to select an appropriate sample. While some of these theories propose random sampling (Allik et al., 2013; Marchant, Simons, \& de Fockert, 2013; Maule \& Franklin, 2016), others suggest non-random factors that can favor specific items for ensemble sampling (Myczek \& Simons, 2008). As the limited-capacity sampling theories are organically related to focused attention, it is natural to suppose that factors guiding attentional deployment in a scene can be used for item prioritization in sampling. As an example, De Fockert and Marchant (2008) demonstrated the role of top-down attentional set towards a smallest or largest item in biasing ensemble reports. Recently, Kanaya, Hayashi, and Whitney (2018) focused on a fundamental role played by bottom-up determinants of focused attention, such as saliency, in item sampling for ensemble perception. In their study, participants judged the mean size or mean temporal frequency (TF) of a set of one, four, eight, or 14 circles. Remarkably, the increased set size caused growth in an overestimation bias in both mean size and mean TF judgments. According to Kanaya et al. (2018), this growing bias can reflect non-random sampling of $\sim \sqrt{ } \mathrm{N}$ most salient items, that is, items with large sizes and high TF. For instance, when four items with different sizes are averaged, the larger half (i.e., $\sqrt{ } 4=2$ ) of the circles are sampled preferentially (or amplified), yielding a slight overestimation bias. When the set size is, say, 16 , only a quarter (i.e., $\sqrt{ } 16=4$ ) of the largest circles are sampled. As soon as the fraction of amplified items decreases as a function of growing set size in accordance with the square root function, there will be no capacity to sample any exemplars except the most salient ones, which should cause a heavier overestimation bias. A similar effect was recently shown in the domain of emotion averaging, suggesting the robustness of attentional amplification in ensemble perception (Goldenberg, Weisz, Sweeny, Cikara, \& Gross, 2020).

Although the systematic effect of set size on average overestimation has been demonstrated in other studies (Price, Kimura, Smith, \& Marshall, 2014; Smith \& Price, 2010), Kanaya et al. (2018) were the first to find evidence that this effect could have to do with non-random attentional sampling of salient items rather than response bias. It is important that the authors called the effect "saliency-based amplification" and not just "sampling." Unlike the straightforward versions of limited-capacity sampling (e.g., Myczek \& Simons, 2008), the idea of amplification is less restrictive and may imply that items beyond the intended sample contribute to mean estimation. Although the use of the term "amplification" is suggestive, Kanaya et al. (2018) did not clearly specify the "fate" of items outside an amplified sample and their contribution to ensemble summaries. Is a subsample of most salient items (e.g., as big as $\sqrt{ } \mathrm{N}$ ) a sole basis of ensemble integration? Or, is this subsample really amplified among the rest of the sampled items, which, nevertheless, are processed altogether and contribute to the ensemble representation? We sought to answer these questions in our study.

\section{Overview of our study}

Agreeing with Kanaya et al.'s (2018) interpretation of the amplification effect as a result of attentional modulation of individual item contributions to ensemble statistics, we used it as a tool to further probe the relationship between attended and unattended elements in ensemble integration. We used Kanaya et al.'s paradigm as a basis for experiments but made some important modifications.

First, to test the contribution of amplified items independently from the contribution of the entire ensemble, we needed to manipulate the distributional properties of salient subsamples separately from the entire ensemble distributions. It could not be done in the original Kanaya et al. (2018) paradigm, because saliency was an inherent property of a to-beaveraged dimension. For example, if large items are always more salient among small ones than vice versa (Treisman \& Gormican, 1988), then the most salient subsample of a size ensemble cannot be changed independently without changing the whole distribution. To deal with this issue, we used two different feature dimensions, one to manipulate saliency and another to create a task-relevant ensemble distribution. The saliency-related feature was size, as in Kanaya et al. (2018). The task-relevant, to-be-averaged dimension was orientation, which, due to its circular nature, is not supposed to inherently bias saliency to one or another part of the distribution. Using these two independent dimensions, we were able to assign higher saliency to elements with any orientations while keeping the whole orientation distribution intact. This allowed us 
to parametrically shift the salient subsample relative to the ensemble mean (Experiment 1) or change its bandwidth (Experiment 2). Another important advantage of this technique was that we were able to compare averaging performance in ensembles with and without obvious saliency cues.

The second important addition compared to Kanaya et al. (2018) was our special focus on the analysis of averaging precision, which we measured as the standard deviation $(S D)$ of the observer's error distributions (note that $S D$ is inversely related to precision). Although Kanaya et al. (2018) also measured averaging precision (slopes of psychometric functions), their main question was whether precision changes with set size. We also asked this question in our experiments. Our critical addition to Kanaya et al.'s (2018) analysis was that we estimated $S D$ as a function of parametric manipulations with salient subsamples. The basic idea underlying this analysis is that averaging precision is highly sensitive to the physical range of averaged features. In general, the greater the ensemble range, the greater the averaging error (Dakin, 2001; Fouriezos et al., 2008; Im \& Halberda, 2013; Marchant et al., 2013; Maule \& Franklin, 2015; Solomon, Morgan, \& Chubb, 2011; Sweeny, Haroz, \& Whitney, 2013; Utochkin \& Tiurina, 2014). This range effect appears particularly useful to test how observers integrate the information about more or less salient items, given that we parametrically manipulated the statistics of a salient subsample of items.

To explain how measuring precision coupled with measuring the bias can shed light on integration and amplification in ensemble perception, consider an example in Fig. 1. Here, an observer is shown a set of four triangles oriented $-30^{\circ},-10^{\circ}$, $10^{\circ}$, and $30^{\circ}$ away from the whole set mean (mean $=0^{\circ}$, range $=60^{\circ}$ ). The $10^{\circ}$ and $30^{\circ}$ triangles are larger and, supposedly, more salient. It is easy to see that this salient subset is both strongly shifted and narrowed relative to the whole set (mean $=20^{\circ}$, range $=20^{\circ}$ ). Three rough predictions about the observer's performance can be made depending on how they utilize more and less salient items. In one extreme case (leftmost prediction panel of Fig. 1), participants naturally pay attention to a more salient group of items and sample their observations exceptionally from that group. This prediction is highly consistent with the strongest version of sampling based on focused attention (Myczek \& Simons, 2008). Here, we expect a large bias towards the mean of the salient subsample and extremely reduced $S D$ of the error distribution. Another prediction is that observers sample the items equally regardless of their saliency (middle prediction panel of Fig. 1). This is consistent with either the totally distributed, parallel mode of sampling (Baek \& Chong, 2020) or with limited-capacity random sampling, but in both cases no amplification is supposed. This mode of sampling predicts the unbiased error distribution with the large $S D$. Finally, the third prediction is that items (either all presented items or a limited group of random items) are sampled from the entire set but more salient (i.e., attended) items get more weight due to attentional amplification (Kanaya et al., 2018, rightmost prediction panel of Fig. 1), since attention is sometimes thought of as a mechanism that increases the relative precision, or "peakiness," of attended representations (Alvarez, 2011; Baek \& Chong, 2020; Lu \& Dosher, 1998). Here, we expect a moderate bias and quite large $S D$, because amplification shifts and narrows the peak part of the distribution but also elongates the tail part.

Using this logic, in Experiment 1, we parametrically manipulated the shift of a narrow-range salient subsample of larger elements relative to the mean. This manipulation was important to test whether there is a systematic effect of saliency. Moreover, we compared averaging performance in such sets with control displays where no external saliency cues were presented, that is when all items were same size. In Experiment 2, we parametrically manipulated the range of the salient subsample keeping the whole ensemble range fixed to further investigate the whole spectrum of effects that salient items might have of ensemble representation. In addition, in Experiment 2 we compared: (1) full-distribution sets consisting of both salient and less salient items whose orientations covered the whole fixed range, and (2) halfdistribution sets consisting only of salient items whose orientations conveyed only subsample range information. This important comparison gave us an estimate of how observers would have averaged if they had integrated information only from the salient group of items.

\section{Experiment 1}

\section{Method}

Participants Twenty-six students of the HSE University (23 females; mean age $=19.9$ years, $S D=1.3$ ) participated in Experiment 1 for course credit. All had normal or correctedto-normal vision, no color deficiency, and no psychological disorders. Before the beginning of the experiment, participants gave written informed consent.

Apparatus and stimuli Stimuli were presented using PsychoPy for Linux (Peirce et al., 2019) on a standard VGA monitor with a refresh frequency of $75 \mathrm{~Hz}$ and a 1,600 $\times$ 1,200-pixel spatial resolution on a homogeneous gray field. The viewing distance was about $50 \mathrm{~cm}$. From that distance, one pixel subtended approximately $0.032^{\circ}$ of visual angle. A $14.87^{\circ} \times 14.87^{\circ}$ square at the center of the screen was used as a "working" field for stimulus presentation.

Stimulus sets consisted of white isosceles triangles varying in size and orientation. The height-to-width ratio of the triangles was 2:1. There could be four, eight, or 16 triangles presented on a screen. The triangles were distributed randomly across 16 cells of an imaginary $4 \times 4$ grid (each cell was $3.7^{\circ} \times$ 


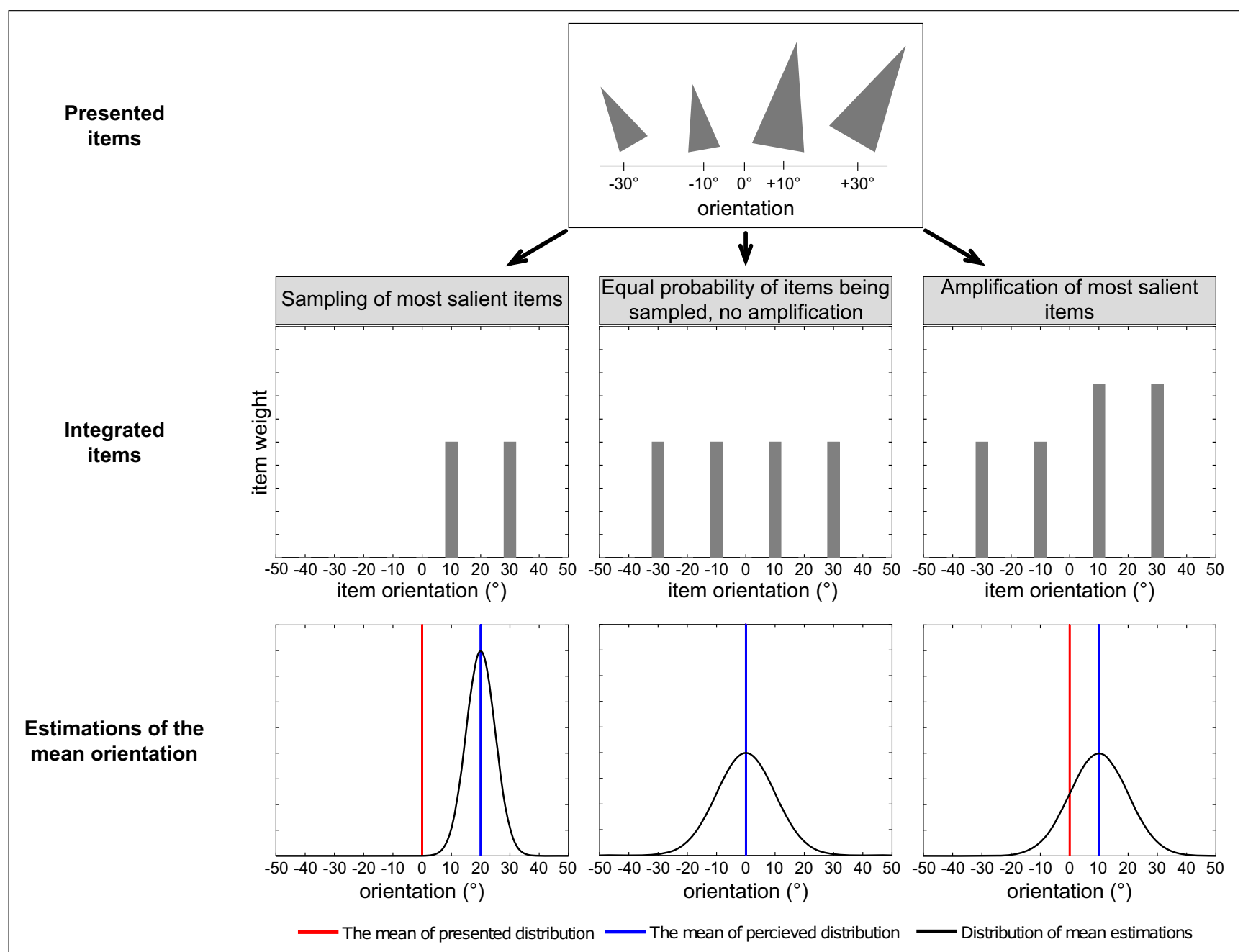

Fig. 1 Hypothetical distributions of mean estimates predicted by different accounts (left to right: only relatively salient items are sampled; items are sampled with equally probability without amplification; items are sampled from the entire set but more salient items get more weight).

$3.7^{\circ}$ in size). Each cell could contain only one triangle (if any) that was centered on the center of the cell with a random jitter within $\pm 0.95^{\circ}$ in both horizontal and vertical dimensions. Orientations of individual triangles were drawn from a uniform distribution. To construct the orientations distribution, we randomly generated the mean between $1^{\circ}$ and $360^{\circ}$ of rotation in each trial. Individual orientations were $-30^{\circ},-10^{\circ}$, $+10^{\circ},+30^{\circ}$ away from that mean. Depending on set size, these four orientations were presented one, two, or four times per set (for set sizes four, eight, and 16, respectively) and were uniformly distributed among items. The actual mean orientation was never presented as a set member.

To manipulate the saliency of the items, we used different sizes. Based on an idea of size as a dimension causing visual search asymmetry (Kanaya et al., 2018; Proulx, 2010; Proulx \& Green, 2011; Treisman \& Gormican, 1988), we used large size to make some of the items more salient and small size to make the rest of the items less salient. To generate the sizes for
The upper row shows an example physical distribution of orientations in a set of items with various salience. The middle row shows items that are sampled and their weights. The bottom row shows expected distributions of mean estimates

each particular display, we randomly chose the mean height of triangles from the interval between $0.63^{\circ}$ and $1.26^{\circ}$ of visual angle. For large items, the mean height was multiplied by 1.3 , and for small items, it was multiplied by 0.7 . The width of the triangles was always scaled in accordance with the height to fit the $2: 1$ ratio.

To systematically manipulate the relationships between the salient subsample and the rest of the set, we assigned large and small sizes to orientations drawn from different parts of the orientation distribution. Overall, we used three sizeorientation distributions: (1) "clockwise," where half of the elements oriented clockwise from the mean (i.e., all $+10^{\circ}$ and $+30^{\circ}$ triangles) were large (Fig. $2 \mathrm{~A}$ ); (2) "counterclockwise," where half of the elements oriented counterclockwise from the mean (i.e., all $-10^{\circ}$ and $-30^{\circ}$ triangles) were large (Fig. 2B); (3) "middle," where medium elements $\left(-10^{\circ}\right.$ and $\left.+10^{\circ}\right)$ were large, whereas extreme elements $\left(-30^{\circ}\right.$ and $\left.+30^{\circ}\right)$ were small (Fig. 2C). It is easy to see that the mean orientation 


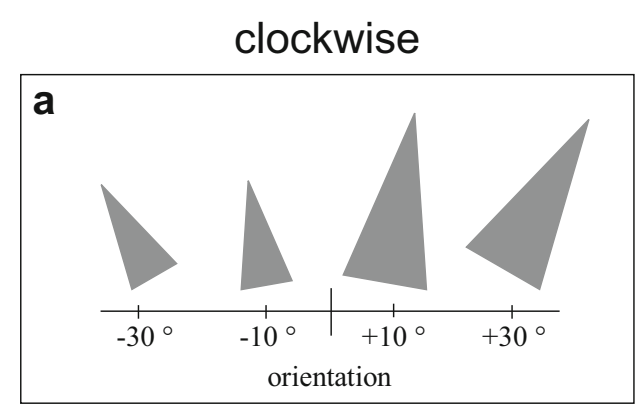

middle

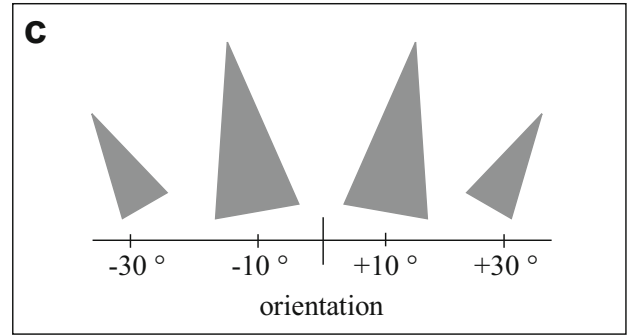

Fig. 2 Four types of size-orientation distributions used in Experiment 1: The mean orientation of bigger items could have (A) more clockwise or (B) more counterclockwise relative to the entire ensemble, or (C) be

of the middle subsample is exactly the same as the mean orientation of the entire set. By contrast, the mean orientations of clockwise and counterclockwise subsamples are strongly shifted (by $20^{\circ}$ in a respective direction) away from the mean. In a fourth, control condition, all items had identical sizes equal to the mean size generated for a given trial (Fig. 2D).

Procedure Each trial started with a fixation cross for $500 \mathrm{~ms}$ followed by a set of triangles that was shown for $500 \mathrm{~ms}$. After that, a randomly oriented test triangle appeared in the center of the screen and remained present until the participant's response confirmation. The test triangle was surrounded by a
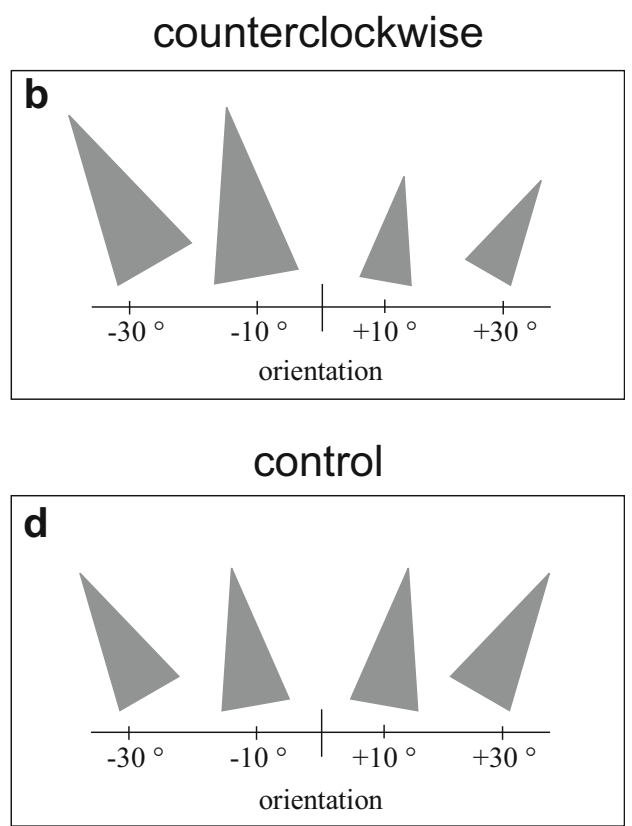

centered in the middle of the entire ensemble. In the control condition (D), all elements had the same size

black adjustment ring with a white slider. The slider location on the ring corresponded to the rotation angle pointed by the apex of the test triangle. Participants were asked to adjust the orientation of the test triangle to match the mean orientation of the just-seen set dragging the slider with mouse clicks on the ring (Fig. 3). No instruction was given about triangles size. When the triangle was adjusted, participants were asked to confirm their response by pressing a spacebar. After doing that, participants were shown a blank screen with an instruction prompting press the spacebar when they are ready to start the next trial, so that they could proceed with the task at a comfortable pace.

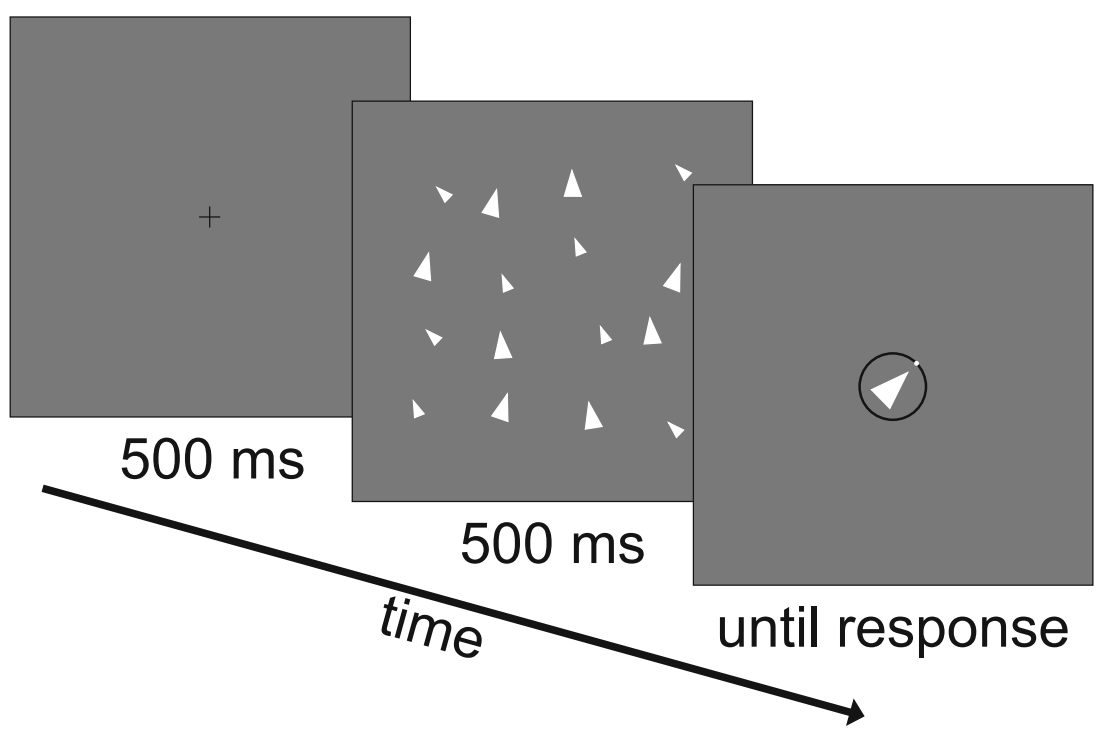

Fig. 3 An example trial of Experiment 1 for set size $=16$ 
Before the experimental block, all participants completed a practice block consisting of 12 trials. Feedback about accuracy was provided after each trial of this block. For the feedback, both the actual mean and adjusted mean orientations were shown on a screen. The experimental block included 600 trials. Trials representing different experimental conditions were run in random order. There was no feedback about response accuracy during the experimental block.

Design and data analysis The experiment had a 4 (saliency distribution: clockwise, counterclockwise, middle, and control) $\times 3$ (set size: four, eight, and 16) within-subject design. Overall, each participant was exposed to four saliency distributions $\times$ three set sizes $\times 50$ repetitions $=600$ trials.

In each trial, we calculated an adjustment error as a difference between observer's response and correct mean orientation $($ Error $=$ Response - Correct) that could range between $-180^{\circ}$ and $180^{\circ}$. Resulting error distributions were used to estimate two parameters: mean as a measure of the systematic Bias and standard deviation $(S D)$ as a measure of representational imprecision. It can be seen from the Error formula that the positive Bias reflects a tendency to adjust the mean orientation more to the clockwise from the correct answer, whereas the negative Bias reflects a tendency to adjust the mean orientation more to the counterclockwise from the correct answer.

\section{Results and discussion}

To reduce the effect of presumably random responses on data analysis, we excluded trials where observers showed an error greater than $\pm 3 S D$ of the overall error distribution, which was approximately $\pm 63^{\circ}$. This led to $0.86 \%$ of trials being excluded from the analysis.

Bias. We found the strong effect of the saliency distribution on the bias in the estimation of the mean orientation $\left(F(3,75)=81.74, p<.001, \eta_{p}^{2}=.64\right)$. Overall, we observed systematic biases towards a subsample of larger items (Fig. 4A). In particular, we noted that observers adjusted the mean orientation with a significant clockwise $\left(M=6.6^{\circ}\right)$ and counterclockwise $\left(M=-8.3^{\circ}\right)$ bias in the corresponding conditions compared to the control condition $(t \mathrm{~s}(25)>7.751, p \mathrm{~s}<.001$, Bonferroni-corrected $\alpha=.008$, Cohen's $d$ 's $>1.52$ ). In the middle saliency distribution, the bias did not differ from the control condition (middle: $M=-1.2^{\circ}$, control: $M=-0.7^{\circ}$; comparison: $t(25)=1.34, p=.19$, Cohen's $\left.d^{\prime}=0.262\right)$. At the same time, the magnitudes of the biases were substantially smaller than those one might predict if observers had sampled only from large items (one-sample comparisons: clockwise, $M=6.6^{\circ}$ vs. predicted $20^{\circ}, t(25)=15.45, p<$ .001 , Cohen's $d^{\prime}=3.03$; counterclockwise, $M=-8.3^{\circ}$ vs. predicted $-20^{\circ}, t(25)=14.00, p<.001$, Cohen's $d^{\prime}=$
2.75). We also found the effect of saliency distribution $\times$ Set size $\left(F(6,150)=3.02, p=.008, \eta_{p}^{2}=.03\right)$. It was provided by the clockwise and counterclockwise conditions, where the bias tended to grow in absolute magnitude as a function of set size $\left(F_{\mathrm{S}}(2,50)>4.64, p \mathrm{~s}<.01\right.$, $\eta_{p}^{2}>$.03). Overall, the biasing effect of large items and its dependence on set size are well in line with the results by Kanaya et al. (2018) and their interpretation in terms of attentional amplification.

Precision. Set size had an effect on the precision of orientation averaging $\left(F(2,50)=38.75, p<.001, \eta_{p}^{2}=0.11\right)$ causing a steady decrease in the $S D$ as a function of set size $(t \mathrm{~s}(25)>2.842, p \mathrm{~s}<.009$, Bonferroni-corrected $\alpha=$ .017 , Cohen's $d$ 's $>0.557$, Figure 4B). Specifically, the effect was observed in all types of distributions $\left(F_{\mathrm{S}}(2,50)\right.$ $>6.08, p \mathrm{~s}<.004$, Bonferroni-corrected $\alpha=.013, \eta_{p}^{2} \mathrm{~s}=$ $.04)$. We also found an effect of the saliency distribution on the $S D\left(F(3,75)=5.95, p=.01, \eta_{p}^{2}=.02\right)$. Specifically, the "middle" saliency distribution yielded a smaller $S D$ compared to other distributions $(t \mathrm{~s}(25)>2.96$, $p \mathrm{~s}<.006$, Bonferroni-corrected $\alpha=.008$, Cohen's $d$ 's $<$ 0.581 ), whereas the rest of the distributions yielded similar $S D$ 's $(t \mathrm{~s}(25)<0.21, p \mathrm{~s}>.83$, Bonferroni-corrected $\alpha$ $=.017$, Cohen's $d$ 's $<0.042$ ).

Although the set size manipulation was introduced, following Kanaya et al. (2018), to test the relative contribution of potentially amplified items among other items to averaging, we did not find evidence that set size-related drop in the $S D$ is specifically caused by the salient subsample. We conclude that based on this finding, this drop was equally observed in all trials containing items of various saliency, as well in the control trials with all items having equal saliency. The generality of this pattern can reflect "noise cancellation" (Baek \& Chong, 2020), whereby averaging precision benefits from the accumulation of multiple individual samples measured with uncorrelated errors (Alvarez, 2011; Galton, 1907).

At the same time, we found one specific effect of a relatively salient subsample on the precision of averaging: The "middle" type of the distribution yielded systematically lower $S D$ s in all set sizes. The amplification of salient items can possibly explain this whole pattern. If amplification biases averaging towards attended items, as Kanaya et al. (2018) suggest, then these amplified items are included into summary statistics with increased weights. In this case, a weighted $S D$ will be smaller when highly weighted items tend to lay in the middle of the distribution than when they tend towards its "tails" or when they are equally weighted. Accordingly, if amplification overweighs large items, then it would reduce the perceived bandwidth of orientations in "middle" displays, resulting in more precise averaging. Note that this explanation necessarily implies that both salient and less salient items are taken into account in averaging. 

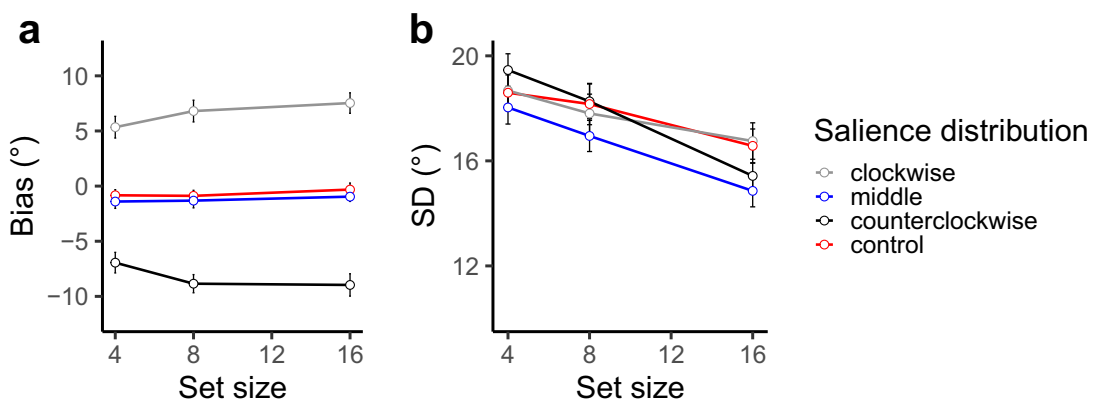

Fig. 4 Results of Experiment 1: (A) bias and (B) standard deviation $(S D)$ of error distributions as a function of set size and the type of size-orientation distribution. Error bars represent the standard error of the mean

Overall, the results of Experiment 1 provide evidence that under independent manipulations of subsample saliency via size, the estimated average orientation was systematically biased towards a subsample of large, presumably more salient items when the subsample mean was shifted away from the overall mean ("clockwise" and "counterclockwise" conditions). When the subsample had the same mean as the whole set but fit in a narrower range ("middle" condition), we observed benefit in the precision of averaging. Both these findings are consistent with overweighting the representations of larger elements that, in turn, might be interpreted in terms of saliency-based amplification (Kanaya et al., 2018). Moreover, we replicated the growth of the bias towards the large subsample as a function of set size. According to the amplification account (Kanaya et al., 2018), this can indicate a disproportionate change in representational weights of more and less salient items due to slowly growing integration capacity (Whitney \& Yamanashi Leib, 2018). On the other hand, our data suggest that the bias and precision change were not as extreme as might be expected from exceptional sampling from the salient subsample.

\section{Experiment 2}

In Experiment 2, we systematically manipulated the range of orientations of the more salient group of triangles, while keeping the overall range fixed. This allows deeper testing of the contribution of attentional amplification in a different way, independent of that used in Experiment 1. In brief, the basic idea of Experiment 2 is that, if larger items are amplified, the precision of averaging should be better if the larger items have middle orientations (e.g., $-10^{\circ}$ and $10^{\circ}$ ), and precision should be worse if the larger items have orientations extremely tilted away from the mean (e.g., $-30^{\circ}$ and $30^{\circ}$ ). Another principal goal of Experiment 2 was to obtain the straightforward baseline performance of an observer sampling strictly from the salient subsample and discount all other items. This was accomplished by introducing the half-distribution set condition where only large-size items were presented matching in orientation range with large-size items from full sets containing both large and small items. The comparison between the half- distribution sets and full-distribution sets matching in the salient half-distribution set range can directly show whether the full range of presented orientations contributes to ensemble averaging.

Participants Twenty-six students of the HSE University (21 females, mean age $=19.8$ years, $S D=1.3$ ) participated in Experiment 2. Ten of these participants also had participated in Experiment 1. All participants were tested as having normal or corrected-to-normal visual acuity and no color vision deficiency, and all reported having no psychological disorders. Before the beginning of the experiment, participants gave written informed consent.

Apparatus and stimuli Our apparatus was the same as in Experiment 1 . We used stimuli with basically the same characteristics as in Experiment 1, except the way we manipulated saliency distributions and the presence of a "non-salient" subsample. For saliency distributions, we made either middle elements $\left(-10^{\circ}\right.$ and $+10^{\circ}$ from the mean) larger ("middle" distribution), or extreme elements $\left(-30^{\circ}\right.$ and $+30^{\circ}$ from the mean) larger ("extreme" distribution). To manipulate the potential availability of the non-salient subsample, we presented our observers with either the full-distribution set or the halfdistribution set displays. In the full-distribution set condition, we had the same set sizes as in Experiment 1 (four, eight, or 16 items), with half of items being large and another half being small. In a second, half-distribution set condition, we presented only the large half of items (four, eight, or 16 in total) and no small items. The full-distribution sets compared to their half-distribution set "counterparts" allowed us to estimate how much averaging of ensembles with both more and less salient elements differs from averaging based solely on highly salient elements. Manipulating the saliency distribution and the presence of a "non-salient" subsample, we had four different distribution structures (Fig. 5): (1) "extreme fulldistribution set" (Fig. 5A), (2) "middle full-distribution set" (Fig. 5B), (3) "extreme half-distribution set" (Fig. 5C), and (4) "middle half-distribution set" (Fig. 5D).

Procedure The procedure was the same as in Experiment 1. 
extreme full-distribution

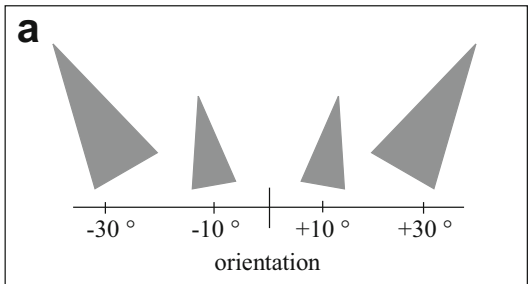

extreme half-distribution

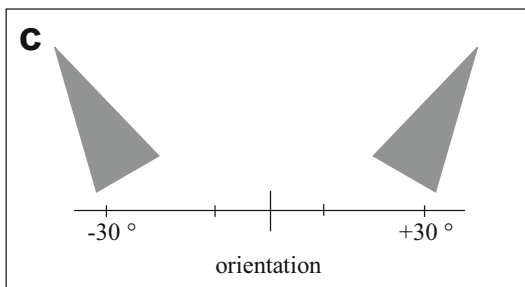

Fig. 5 Four types of distribution structures used in Experiment 2: The mean orientation of bigger items could have (A) extreme orientations while smaller items could have middle orientations, (B) middle

Design and data analysis The experiment had a 4 (Distribution structure: extreme full-distribution set, middle full-distribution set, extreme half-distribution set, middle half-distribution set) $\times 3$ (set size: four, eight, and 16) within-subject design. Overall, each participant was exposed to four distribution structures $\times$ three set sizes $\times 50$ repetitions $=600$ trials. As in Experiment 1, we analyzed parameters of the error distributions in orientation averaging. However, here we were focused on the $S D$ as a principal dependent variable. Since our distribution manipulations in this experiment kept the mean of all possible subsamples physically the same, we did not expect the bias to be an informative variable.

\section{Results and discussion}

One percent of trials were excluded from the analysis based on the same exclusion criterion as in Experiment 1. The precision of orientation averaging was strongly affected by the distribution structure $\left(F(3,75)=168.30, p<.001, \eta_{p}^{2}=.45\right)$. Specifically, in the full-distribution set, the error $S D$ tended to increase as the range of a more salient subsample (large items) became larger between the middle and extreme versions of this subsample (middle: $M=16.9^{\circ}$, extreme: $M=$ $19.5^{\circ}$, comparison: $t(25)=7.093, p<.001$, Bonferronicorrected $\alpha=.008$, Cohen's $d=1.39$ ). The same pattern was found for the half-distribution set consisting only of large items: Namely, the $S D$ in the middle half-distribution set was smaller than the $S D$ in the extreme half-distribution set (middle: $M=13.4^{\circ}$, extreme: $M=23^{\circ}$, comparison: $t(25)=17.269$, $p<.001$, Bonferroni-corrected $\alpha=.008$, Cohen's $d=3.39$ ). Critically, this pattern in the half-distribution set displays was an exaggerated pattern found in their full-distribution set middle full-distribution

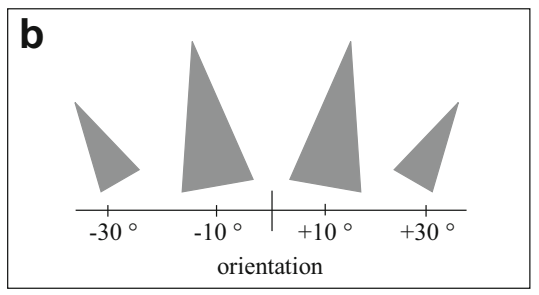

middle half-distribution

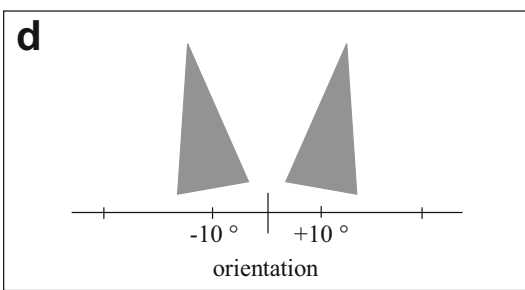

orientations while smaller items could have extreme orientations, $(\mathbf{C})$ only extreme orientations, while small items were not shown, or (D) only middle orientations, while small items were not shown

counterparts (Fig. 6). That is, the middle half-distribution set $S D$ was lower than the middle full-distribution set $S D(t(25)=$ 9.444, $p<.001$, Bonferroni-corrected $\alpha=.008$, Cohen's $d=$ $1.852)$, whereas the extreme half-distribution set $S D$ was greater than the full-distribution set $(t(25)=10.157, p<$ .001 , Bonferroni-corrected $\alpha=.008$, Cohen's $d=1.992$ ). In other words, the half-distribution sets produced dramatic range effects correlated with physically different ranges of presented orientations. In full-distribution sets where the overall ranges were same, the $S D$ s also changed in a direction predicted by the range of a large (more salient) subsample, but their changes were attenuated by the presence of a small (less salient) subsample.

Similar to Experiment 1, we observed the effect of set size on the error $S D\left(F(2,50)=11.51, p<.001, \eta_{p}^{2}=.03\right)$ indicating that the $S D$ tends to decrease as a function of set size (Fig. $6)$. We also found a small effect of distribution structure and set size $\left(F(6,150)=2.33, p=.032, \eta_{p}^{2}=.011\right)$. This $S D$ decrement was mainly observed in the middle fulldistribution and middle half-distribution $(F(2,50)>6.95, p<$

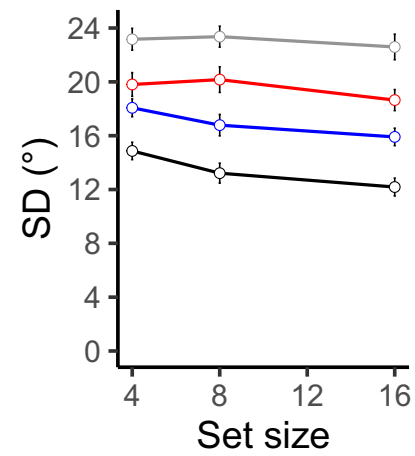

Distribution structure

$\circ$ middle full-distribution

- extreme full-distribution

- middle half-distribution

extreme half-distribution

Fig. 6 Averaged standard deviation $(S D)$ for each set size and distribution structure condition. Error bars represent standard error of the mean 
.002 , Bonferroni-corrected $\alpha=.013, \eta_{p}^{2}>.06$ ) and not in the extreme full-distribution and half-distribution $(F(2,50)<2.77$, $p>.072, \eta_{p}^{2}<.02$ ).

An additional analysis was run to test whether the changes in the distribution type caused any specific biases in average estimates. As expected from the symmetric distributions of both large and small items in all conditions, we did not observe any specific effect of the distribution structure $(\mathrm{F}(3,75)=$ $\left.0.1, \mathrm{p}=.96, \eta_{p}^{2}=.001\right)$, or set size $(\mathrm{F}(2,50)=0.089, \mathrm{p}=.914$, $\left.\eta_{p}^{2}=.0004\right)$, or their interaction $\left(\mathrm{F}(6,150)=2.13, \mathrm{p}=.052, \eta_{p}^{2}\right.$ $=.03$ ).

Overall, the results of Experiment 2 showed that the range of a relatively salient subsample (large items) modulates the precision of the estimated mean orientation in a set including both large and small items. This is in line with an idea of subsample amplification driven by selective attention towards this subsample (Kanaya et al., 2018). However, the experiment also demonstrated that the orientation distribution of salient items does not fully determine the precision, as the range effect of the salient subsample was attenuated by the presence of less salient items in the full-distribution condition compared to the half-distribution condition. Therefore, Experiment 2 provides additional evidence that set members beyond the subsample of potentially amplified more salient items are also included in an ensemble representation.

In addition, we observed the growth of the precision as a function of set size. However, unlike Experiment 1, this growth of the precision was not consistent across conditions. Specifically, precision did not benefit from the larger set sizes in the "extreme" conditions that yielded greater absolute $S D$ s. One possible explanation is that it is high overall noise in averaging that makes the relatively small effect of noise cancellation poorly visible. This speculation will be addressed in the General discussion in the broader context of inconsistent reports of set size effects on averaging precision in the literature.

\section{General discussion}

In this study, we investigated the contribution of individual items into the global ensemble representation as a function of their saliency. Specifically, we addressed the recently suggested amplification theory (Kanaya et al., 2018), emphasizing the systematically differential contribution of various items into averaging as a function of their saliency. Since we separated saliency-defining manipulations according to size from statistical properties of the overall to be averaged stimulus dimension (orientation), we were able to probe the effects of amplified subsamples independently of the effects of the full-range distributions.

In sum, our results across the two experiments consistently showed the patterns in favor of the amplified contributions of larger items, even though the size itself was not a relevant feature. We demonstrated it in two different ways - as a systematic bias in orientation averaging when a salient subdistribution is shifted relative to the superset mean (Experiment 1) and as a systematic change in the precision when a salient distribution changes its range within the constantly wide overall distribution (Experiment 2). Therefore, we basically replicated evidence for size amplification in ensemble perception from Kanaya et al. (2018). That amplification has its effect not only on the bias but also on precision, as well as the cross-dimensional transfer of amplification to orientation averaging, are important extensions of Kanaya et al.'s (2018) findings.

The growth of averaging precision with set size as observed in our study is not always reported in the literature. Some studies have found precision to be stable across set sizes larger than four (Ariely, 2001; Chong \& Treisman, 2005b; Haberman \& Whitney, 2010; Kanaya et al., 2018; Tokita et al., 2016), while other studies have found evidence for steady improvement in larger set sizes (Baek \& Chong, 2020; Lee, Baek, \& Chong, 2016; Parkes et al., 2001; Robitaille \& Harris, 2011). The lack of set size effect on precision can indicate either that a limited number of items are sampled (Haberman \& Whitney, 2010; Kanaya et al., 2018; but see Utochkin \& Tiurina, 2014 for some logical counterarguments) or that the advantage of noise cancellation is eliminated by the growing noise in each individual representation resulting from the increased set size (Alvarez, 2011). It is possible, therefore, that whether the benefit from larger set sizes is observable may depend on how quickly set size effects are accumulated in noise cancellation and in individual representational noise. That is, if the strength of noise cancellation grows faster than the individual representational noise, we might expect a growth in averaging precision. Also, depending on particular stimulus parameters, the benefit from noise cancellation can be observed in some experiments and not observed in others. For example, if individual features are highly variable, the contribution of noise cancellation to the averaging error can be relatively small compared to the contribution of stimulus variance. This latter suggestion could explain the inconsistency of the set size effect on precision in different conditions of our Experiment 2, where precision did not change with set size in more "extreme" displays but it tended to improve in more "middle" displays. However, this suggestion needs further empirical testing.

Yet, our critical finding was evidence that both large and small items influence ensemble averaging, as was revealed by the range effects on the error $S D$ (Dakin, 2001; Fouriezos et al., 2008; Im \& Halberda, 2013; Marchant et al., 2013; Maule \& Franklin, 2015; Solomon et al., 2011; Sweeny et al., 2013; Utochkin \& Tiurina, 2014). Experiment 1 showed that the $S D$ did not change or changed only slightly in the presence of a salient subsample compared to the control 
displays with no salient subsamples, even though the subsamples were half as wide as the entire set range. In addition, Experiment 2 showed how the presence of low salient items (full-distribution set trials) compensates for the dramatic range of effects from the more salient items presented alone (halfdistribution set trials). When the large items are extremely remote from the mean, the addition of small items in the middle of the distribution reduces the error (Fig. 6). Conversely, when the large items are in the middle, the addition of small ones at the extremes increases the error (Fig. 6). This latter finding is especially interesting, as it shows that observers cannot attend to salient items alone and completely ignore less salient items, even when ignoring is the best strategy. Indeed, if all large, salient items are so close to the mean and all small items are so remote it is beneficial to compute the mean orientation based only on large items that grab attention preferentially. However, our results suggest that observers cannot ignore small items at extremes, even despite the lack of saliency and reliability for mean estimation.

The large theoretical question the amplification effect aimed to clarify in Kanaya et al.'s (2018) original study concerned basic mechanisms of ensemble integration from individual representations. In particular, according to Kanaya et al. (2018), amplification can involve the efficiently integrated set size that grows slower than the presented set size $(\sim \sqrt{ } \mathrm{N}$, Whitney \& Yamanashi Leib, 2018). Adopting the attentional nature of amplification suggested by Kanaya et al. (2018), this " $\sqrt{\mathrm{N}}$ account" appears to be very consistent with the limited capacity of attentional processing (Gorea et al., 2014; Pylyshyn \& Storm, 1988; Scholl, 2001). Does this mean that people pick the $\sqrt{\mathrm{N}}$ items and that saliency completely determines which particular items are picked? Our results suggest that this is definitely not the case, since averaging performance was very much affected by the less salient, small items (see also Oriet \& Brand, 2013).

There are at least three theoretical scenarios that could account for both the increasing bias and noise cancellation effect. In the first scenario, saliency-based amplification increases the probability of a particular orientation being sampled for subsequent averaging among the limited sample elements. It is also implied by this scenario that the number of integrated items grows with set size, which would explain the growth in precision (noise cancellation; Baek \& Chong, 2020), and that this number grows more slowly than set size (e.g., as $\sim \sqrt{ } \mathrm{N}$ ), which would explain the increased bias toward salient items, since the chance for a less salient item to be sampled decreases (Kanaya et al., 2018). The second scenario suggests that amplification increases the weight of more salient items and decreases the weight of less salient items in a set, although the subset itself is sampled randomly (note that in this case, sampling precedes amplification, otherwise it is not random). Like in the first scenario, the sample size should increase with the physical set size to provide noise cancellation. In the third scenario, all items from the whole ensemble are integrated and a limited subsample is additionally amplified due to its greater saliency. More generally, this scenario implies that the visual system builds an ensemble representation based on the overall impression of all visible items (Oriet \& Brand, 2013) and adjusts it based on a few items happening to be attended to (Baek \& Chong, 2020). Obviously, it predicts noise cancellation. In addition, the amplified sample should also grow with set size to account for the increasing bias. Our data do not allow us to dissociate between these scenarios and this should be addressed in future research.

In summary, our study aimed to probe the "fate" of individual item representations in the computation of an ensemble mean feature. In line with some of the previous studies, we found that the reported average orientation is more greatly affected by a subset of relatively salient items (the amplification effect, Kanaya et al., 2018), but contributions from the rest of the items are also present. Therefore, we conclude that saliency is an important but not an absolute determinant of what is efficiently integrated for an ensemble percept. In addition, we demonstrated that averaging precision tended to grow with set size (probably as a result of noise cancellation; Baek \& Chong, 2020), which suggests that the effective set size increases with the number of items in an ensemble.

Acknowledgements This research was implemented within the Basic Research Program of the National Research University Higher School of Economics (TZ-60 in 2020). The authors would like to thank Sang Chul Chong and an anonymous reviewer for helpful comments on an earlier draft of the article.

Open practices statement The data and materials for all experiments are available at the Open Science Framework (https://osf.io/e5bg2/), and none of the experiments were preregistered.

Author contributions A.I. prepared experimental scripts, ran experiments, analyzed data, and contributed to writing the manuscript. I.U. conceptualized the study, designed experiments, and contributed to writing the manuscript.

\section{References}

Allik, J., Toom, M., Raidvee, A., Averin, K., \& Kreegipuu, K. (2013). An almost general theory of mean size perception. Vision Research, 83, 25-39. https://doi.org/10.1016/j.visres.2013.02.018

Alvarez, G. A. (2011). Representing multiple objects as an ensemble enhances visual cognition. Trends in Cognitive Sciences, 15(3), 122-131. https://doi.org/10.1016/j.tics.2011.01.003

Alvarez, G. A., \& Oliva, A. (2009). Spatial ensemble statistics are efficient codes that can be represented with reduced attention. Proceedings of the National Academy of Sciences of the United States of America, 106(18), 7345-7350. https://doi.org/10.1073/ pnas.0808981106

Ariely, D. (2001). Seeing Sets: Representation by Statistical Properties. Psychological Science, 12(2), 157-162. JSTOR. 
Attarha, M., Moore, C. M., \& Vecera, S. P. (2014). Summary statistics of size: Fixed processing capacity for multiple ensembles but unlimited processing capacity for single ensembles. Journal of Experimental Psychology: Human Perception and Performance, 40(4), 1440. https://doi.org/10.1037/a0036206

Baek, J., \& Chong, S. C. (2020). Distributed attention model of perceptual averaging. Attention, Perception, \& Psychophysics, 82(1), 63 79. https://doi.org/10.3758/s13414-019-01827-z

Bronfman, Z. Z., Brezis, N., Jacobson, H., \& Usher, M. (2014). We See More Than We Can Report: "Cost Free" Color Phenomenality Outside Focal Attention. Psychological Science, 25(7), 13941403. https://doi.org/10.1177/0956797614532656

Chong, S. C., \& Evans, K. K. (2011). Distributed versus focused attention (count vs estimate). WIREs Cognitive Science, 2(6), 634-638. https://doi.org/10.1002/wcs.136

Chong, S. C., \& Treisman, A. (2003). Representation of statistical properties. Vision Research, 43(4), 393-404. https://doi.org/10.1016/ S0042-6989(02)00596-5

Chong, S. C., \& Treisman, A. (2005a). Attentional spread in the statistical processing of visual displays. Perception \& Psychophysics, 67(1), 1-13. https://doi.org/10.3758/BF03195009

Chong, S. C., \& Treisman, A. (2005b). Statistical processing: Computing the average size in perceptual groups. Vision Research, 45(7), 891900. https://doi.org/10.1016/j.visres.2004.10.004

Chong, S. C., Joo, S. J., Emmanouil, T.-A., \& Treisman, A. (2008), Statistical processing: Not so implausible after all. Perception \& Psychophysics, 70(7), 1327-1334. https://doi.org/10.3758/PP.70.7. 1327

Cohen, M. A., Dennett, D. C., \& Kanwisher, N. (2016). What is the Bandwidth of Perceptual Experience? Trends in Cognitive Sciences, 20(5), 324-335. https://doi.org/10.1016/j.tics.2016.03. 006

Dakin, S. C. (2001). Information limit on the spatial integration of local orientation signals. JOSA A, 18(5), 1016-1026. https://doi.org/10. 1364/JOSAA.18.001016

Dakin, S. C., \& Watt, R. J. (1997). The computation of orientation statistics from visual texture. Vision Research, 37(22), 3181-3192. https://doi.org/10.1016/S0042-6989(97)00133-8

De Fockert, J. W., Marchant, A. P. (2008) Attention modulates set representation by statistical properties. Perception \& Psychophysics, 70(5), 789-794.

Gardelle, V. de, \& Summerfield, C. (2011). Robust averaging during perceptual judgment. Proceedings of the National Academy of Sciences, 108(32), 13341-13346. https://doi.org/10.1073/pnas. 1104517108

Fischer, J., \& Whitney, D. (2011). Object-level visual information gets through the bottleneck of crowding. Journal of Neurophysiology, 106(3), 1389-1398. https://doi.org/10.1152/jn.00904.2010

Fouriezos, G., Rubenfeld, S., \& Capstick, G. (2008). Visual statistical decisions. Perception \& Psychophysics, 70(3), 456-464. https:// doi.org/10.3758/PP.70.3.456

Galton, F. (1907). Vox Populi [News]. Nature https://doi.org/10.1038/ 075450a0

Goldenberg, A., Weisz, E., Sweeny, T., Cikara, M., \& Gross, J. (2020). The crowd emotion amplification effect [Preprint]. Open Science Framework. https://doi.org/10.31219/osf.io/cn6qy

Gorea, A., Belkoura, S., \& Solomon, J. A. (2014). Summary statistics for size over space and time. Journal of Vision, 14(9), 22. https://doi. org/10.1167/14.9.22

Haberman, J., \& Whitney, D. (2007). Rapid extraction of mean emotion and gender from sets of faces. Current Biology, 17(17), R751R753. https://doi.org/10.1016/j.cub.2007.06.039

Haberman, J., \& Whitney, D. (2009). Seeing the mean: Ensemble coding for sets of faces. Journal of Experimental Psychology: Human Perception and Performance, 35(3), 718-734. https://doi.org/10. 1037/a0013899
Haberman, J., \& Whitney, D. (2010). The visual system discounts emotional deviants when extracting average expression. Attention, Perception, \& Psychophysics, 72(7), 1825-1838. https://doi.org/ 10.3758/APP.72.7.1825

Haberman, J., Harp, T., \& Whitney, D. (2009). Averaging facial expression over time. Journal of Vision, 9(11), 1. https://doi.org/10.1167/ 9.11 .1

Haberman, J., Lee, P., \& Whitney, D. (2015). Mixed emotions: Sensitivity to facial variance in a crowd of faces. Journal of Vision, 15(4), 16. https://doi.org/10.1167/15.4.16

Im, H. Y., \& Halberda, J. (2013). The effects of sampling and internal noise on the representation of ensemble average size. Attention, Perception, \& Psychophysics, 75(2), 278-286. https://doi.org/10. 3758/s13414-012-0399-4

Kanaya, S., Hayashi, M. J., \& Whitney, D. (2018). Exaggerated groups: Amplification in ensemble coding of temporal and spatial features. Proceedings of the Royal Society B: Biological Sciences, 285(1879), 20172770. https://doi.org/10.1098/rspb.2017.2770

Khvostov, V. A., \& Utochkin, I. S. (2019). Independent and parallel visual processing of ensemble statistics: Evidence from dual tasks. Journal of Vision, 19(9), 3. https://doi.org/10.1167/19.9.3

Lee, H., Baek, J., \& Chong, S. C. (2016). Perceived magnitude of visual displays: Area, numerosity, and mean size. Journal of Vision, 16(3), 12. https://doi.org/10.1167/16.3.12

Leib, A. Y., Kosovicheva, A., \& Whitney, D. (2016). Fast ensemble representations for abstract visual impressions. Nature Communications, 7, 13186. https://doi.org/10.1038/ncomms13186

Lu, Z.-L., \& Dosher, B. A. (1998). External noise distinguishes attention mechanisms. Vision Research, 38(9), 1183-1198. https://doi.org/10. 1016/S0042-6989(97)00273-3

Marchant, A. P., Simons, D. J., \& de Fockert, J. W. (2013). Ensemble representations: Effects of set size and item heterogeneity on average size perception. Acta Psychologica, 142(2), 245-250. https:// doi.org/10.1016/j.actpsy.2012.11.002

Maule, J., \& Franklin, A. (2015). Effects of ensemble complexity and perceptual similarity on rapid averaging of hue. Journal of Vision, 15(4), 6. https://doi.org/10.1167/15.4.6

Maule, J., \& Franklin, A. (2016). Accurate rapid averaging of multihue ensembles is due to a limited capacity subsampling mechanism. JOSA A, 33(3), A22-A29. https://doi.org/10.1364/JOSAA.33. 000A22

Morgan, M., Chubb, C., \& Solomon, J. A. (2008). A 'dipper' function for texture discrimination based on orientation variance. Journal of Vision, 8(11), 9. https://doi.org/10.1167/8.11.9

Myczek, K., \& Simons, D. J. (2008). Better than average: Alternatives to statistical summary representations for rapid judgments of average size. Perception \& Psychophysics, 70(5), 772-788. https://doi.org/ 10.3758/PP.70.5.772

Oriet, C., \& Brand, J. (2013). Size averaging of irrelevant stimuli cannot be prevented. Vision Research, 79, 8-16. https://doi.org/10.1016/j. visres.2012.12.004

Parkes, L., Lund, J., Angelucci, A., Solomon, J. A., \& Morgan, M. (2001). Compulsory averaging of crowded orientation signals in human vision. Nature Neuroscience, 4(7), 739-744.

Peirce, J., Gray, J. R., Simpson, S., MacAskill, M., Höchenberger, R., Sogo, H., Kastman, E., \& Lindeløv, J. K. (2019). PsychoPy2: Experiments in behavior made easy. Behavior Research Methods, 51(1), 195-203. https://doi.org/10.3758/s13428-018-01193-y

Price, P. C., Kimura, N. M., Smith, A. R., \& Marshall, L. D. (2014). Sample size bias in judgments of perceptual averages. Journal of Experimental Psychology: Learning, Memory, and Cognition, 40(5), 1321-1331. https://doi.org/10.1037/a0036576

Proulx, M. J. (2010). Size Matters: Large Objects Capture Attention in Visual Search. PLoS ONE, 5(12). https://doi.org/10.1371/journal. pone. 0015293 
Proulx, M. J., \& Green, M. (2011). Does apparent size capture attention in visual search? Evidence from the Müller-Lyer illusion. Journal of Vision, 11(13), 21. https://doi.org/10.1167/11.13.21

Pylyshyn, Z. W., \& Storm, R. W. (1988). Tracking multiple independent targets: Evidence for a parallel tracking mechanism. Spatial Vision, 3(3), 179-197. https://doi.org/10.1163/156856888X00122

Robitaille, N., \& Harris, I. M. (2011). When more is less: Extraction of summary statistics benefits from larger sets. Journal of Vision, 11(12), 18. https://doi.org/10.1167/11.12.18

Rosenholtz, R. (2011). What your visual system sees where you are not looking. Human Vision and Electronic Imaging XVI, 7865, 786510. https://doi.org/10.1117/12.876659

Rosenholtz, R. (2016). Capabilities and Limitations of Peripheral Vision. Annual Review of Vision Science, 2(1), 437-457. https://doi.org/10. 1146/annurev-vision-082114-035733

Scholl, B. J. (2001). Objects and attention: The state of the art. Cognition, 80(1), 1-46. https://doi.org/10.1016/S0010-0277(00)00152-9

Smith, A. R., \& Price, P. C. (2010). Sample size bias in the estimation of means. Psychonomic Bulletin \& Review, 17(4), 499-503. https:// doi.org/10.3758/PBR.17.4.499

Solomon, J. A. (2010). Visual discrimination of orientation statistics in crowded and uncrowded arrays. Journal of Vision, 10(14), 19. https://doi.org/10.1167/10.14.19

Solomon, J. A., Morgan, M., \& Chubb, C. (2011). Efficiencies for the statistics of size discrimination. Journal of Vision, 11(12), 13. https://doi.org/10.1167/11.12.13

Suárez-Pinilla, M., Seth, A. K., \& Roseboom, W. (2018). Serial dependence in the perception of visual variance. Journal of Vision, 18(7), 4. https://doi.org/10.1167/18.7.4
Sweeny, T. D., Haroz, S., \& Whitney, D. (2013). Perceiving group behavior: Sensitive ensemble coding mechanisms for biological motion of human crowds. Journal of Experimental Psychology: Human Perception and Performance, 39(2), 329-337. https://doi.org/10. 1037/a0028712

Tokita, M., Ueda, S., \& Ishiguchi, A. (2016). Evidence for a Global Sampling Process in Extraction of Summary Statistics of Item Sizes in a Set. Frontiers in Psychology, 7. https://doi.org/10.3389/ fpsyg.2016.00711

Treisman, A. (2006). How the deployment of attention determines what we see. Visual Cognition, 14(4-8), 411-443. https://doi.org/10. 1080/13506280500195250

Treisman, A., \& Gormican, S. (1988). Feature analysis in early vision: Evidence from search asymmetries. Psychological Review, 95(1), 15. https://doi.org/10.1037/0033-295X.95.1.15

Utochkin, I. S., \& Tiurina, N. A. (2014). Parallel averaging of size is possible but range-limited: A reply to Marchant, Simons, and De Fockert. Acta Psychologica, 146, 7-18. https://doi.org/10.1016/j. actpsy.2013.11.012

Whitney, D., \& Yamanashi Leib, A. (2018). Ensemble Perception. Annual Review of Psychology, 69(1), 105-129. https://doi.org/10. 1146/annurev-psych-010416-044232

Publisher's note Springer Nature remains neutral with regard to jurisdictional claims in published maps and institutional affiliations. 